\title{
BMJ Open Reporting of exercise dose and dosage and outcome measures for gaze stabilisation in the literature: a scoping review
}

Keith R Cole (1) , ${ }^{1}$ Karen Goodman, ${ }^{1}$ Lena Volland ${ }^{2}$

To cite: Cole KR, Goodman K, Volland L. Reporting of exercise dose and dosage and outcome measures for gaze stabilisation in the literature: a scoping review. BMJ Open 2022;12:e049560. doi:10.1136/ bmjopen-2021-049560

- Prepublication history and additional supplemental material for this paper are available online. To view these files, please visit the journal online (http://dx.doi.org/10.1136/ bmjopen-2021-049560).

Received 27 January 2021 Accepted 17 January 2022
D) Check for updates

(C) Author(s) (or their employer(s)) 2022. Re-use permitted under CC BY-NC. No commercial re-use. See rights and permissions. Published by BMJ.

${ }^{1}$ Health, Human Function, and Rehabilitation Science, The George Washington University, Washington, DC, USA

${ }^{2}$ Department of Clinical Research and Leadership, The George Washington University, Washington, DC, USA

Correspondence to

Dr Keith R Cole;

keithcole@gwu.edu

\section{ABSTRACT}

Objectives The concept of this review is to examine and quantify the reporting of parameters of dose (duration, speed, head excursion) and dosage (daily and weekly frequency, duration) for gaze stabilisation exercises and to report on outcome measures used to assess change in gaze stabilisation following intervention. This review includes any population completing gaze stabilisation exercises.

Design Scoping review.

Methods We searched key terms in the following databases: PubMed, CINAHL, Scopus and Cochrane. Two researchers reviewed titles, abstracts and full-text articles for inclusion. Data retrieved included: patient diagnosis, specific interventions provided, dose and dosage of gaze stabilisation interventions and outcome measures. Results From the initial 1609 results, 138 studies were included. Data extraction revealed that only 13 studies $(9.4 \%)$ reported all parameters of dose and dosage. Most studies used other interventions in addition to gaze stabilisation exercises. Half of the studies did not use a clinical or instrumented outcome measure of gaze stability, using only patient-reported outcome measures. Clinical tests of gaze stability were used in $21.1 \%$ of studies, and instrumented measures of gaze stability were used in $14.7 \%$ of studies.

Conclusions Full reporting of the dose and dosage of gaze stabilisation interventions is infrequent, impairing the ability to translate current evidence into clinical care. Most studies did not use a clinical or instrumented measure of gaze stabilisation as outcome measures, questioning the validity of intervention effects. Improved reporting and use of outcome measures are necessary to establish optimal intervention parameters for those with gaze stability impairments.

\section{INTRODUCTION}

Vestibular input is necessary for accurate gaze stabilisation, spatial orientation during movement and balance. "When vestibular function declines or is lost completely, the ability to maintain gaze during movement and the ability to maintain postural control decreases. These changes increase an individual's risk of falls. ${ }^{2}$ Altered vestibular function may result from many different processes.

\section{Strengths and limitations of this study}

- Inclusion of all populations where gaze stability exercises were used.

- Independent content expert reviewer agreement on inclusion and exclusion criteria at each step of the review process.

Limited to publications in English.

Limited to availability of full text.

As we age, there is a progressive decrease in the number and function of hair cells in the inner ear causing a gradual decline in the reliability of information from the vestibular system. ${ }^{3}$ For some individuals, partial or complete loss in vestibular function occurs in one or both ears due to trauma, toxins, infections, genetics, neurodegeneration or unknown causes. ${ }^{4}$ These pathologic events can occur in individuals of all ages, including children. ${ }^{156}$ A recent epidemiologic study from Germany found that the age-adjusted prevalence of unilateral or bilateral vestibular hypofunction $(\mathrm{VH})$ in the general population is $6.7 \% .{ }^{1}$ Data from the 2008 US National Health Interview survey showed that $14.84 \%$ of respondents had experienced dizziness or balance problems in the last year. ${ }^{6}$ Vestibular dysfunction has large consequences, causing decreases in socioeconomic status and quality of life including changes in driving habits, social isolation and decreased activities of daily living. ${ }^{16}$

Treatment of VH, whether from illness or ageing, includes pharmacologic, surgical and rehabilitative measures. Components of vestibular rehabilitation may involve habituation activities, vestibulo-ocular exercises, optokinetic exercises and balance and gait training. ${ }^{78}$ Gaze stabilisation is the ability to maintain an object on the fovea of the retina (visual focus) during head movements. When gaze stabilisation is impaired, this can create 
visual blurring, dizziness and disequilibrium. ${ }^{9}$ Impairments in gaze stability can be treated with physical therapy interventions that adjust the gain of the vestibulo-ocular reflex. ${ }^{10}$ These vestibulo-ocular exercises vary the speed of head movement, background, visual focal point and plane of head movement while attempting to maintain focus on an object. ${ }^{10}$ Gain can also be improved through the use of movement of the visual field during optokinetic and habituation exercises, especially if the head and neck are rotating. ${ }^{811}$

Several larger systematic reviews have reported the benefits of vestibular rehabilitation, including the use of gaze stabilisation exercises in the treatment of individuals with unilateral peripheral VH. ${ }^{72}$ Despite the common usage of these exercises, a recent clinical practice guideline for peripheral $\mathrm{VH}$ cited level of evidence for gaze stabilisation exercise dosage as only at that of expert opinion. ${ }^{12}$ We suspect that limited reporting of intervention parameters and limited use of outcome measures specific to gaze stabilisation across diagnoses are significant contributing factors.

\section{Review questions}

The purpose of this scoping review was to compile information related to dose and dosage of gaze stabilisation exercises. We suspect that different dosages may be beneficial for different types of patients based on the pathophysiology and chronicity of their dysfunction, but it is our suspicion that reporting of dose and dosage is insufficient for exercise replication and metaanalysis. Further, we intend to discern if measures of change in gaze stability are being reported and used in order to establish effectiveness of recommended dose and dosages of each intervention. This would allow conclusions to be drawn regarding patient improvement following interventions provided. Scoping the literature will identify gaps related to implementation of gaze stabilisation exercise to guide future research. Our research questions are as follows:

- What information related to dose (duration, speed, head excursion) and dosage (daily and weekly frequency, duration) of gaze stabilisation exercises is reported in the literature?

- When a gaze stabilisation intervention is prescribed, what outcome measures are used to assess changes in gaze stabilisation?

\section{METHODS}

We developed our methodology based on the Preferred Reporting Items for Systematic Reviews and Meta-Analyses Extension for Scoping Reviews guidelines and checklist as well as other key works regarding methodology for scoping reviews. ${ }^{13-15}$ The protocol for this scoping review was not registered. Two primary researchers developed the research questions and search criteria. A third researcher assisted with resolution of disagreements and data extraction.

\section{Patient and public involvement}

Patients were not involved in the development, design or execution of this study.

\section{Search strategy}

We developed a list of key search terms from our research question and conducted an initial search using those terms in PubMed and CINAHL. From this, we revised our search terms based on keywords and MESH terms from those articles. A sample of our final search strategy for each database can be found in online supplemental appendix 1 . We used the following databases to complete our search: PubMed, CINAHL, Scopus and Cochrane. Searches were conducted between April 2019 and May 2020. Results from the database searches were exported into Endnote (V.X8.2) and duplicates were removed. Two independent reviewers examined the titles and abstracts of the remaining articles and applied the inclusion and exclusion criteria. Full text for each of the remaining articles was acquired for further review and determination of eligibility. If full text was not available, a notation was made and the study was not included. One hundred thirty-eight articles met all criteria and were used for data extraction.

\section{Inclusion/exclusion criteria}

Our population was humans who performed any gaze stabilisation intervention (such as X1 or X2 exercises or optokinetic exercises). The exercise could be performed as part of an intervention to address vestibular dysfunction or could be used to determine the ability to alter components of gaze stability whether or not vestibular dysfunction was present. However, we excluded individuals with the diagnosis of benign paroxysmal positional vertigo as individuals with this diagnosis do not typically present with impairments of gaze stabilisation. We included all age ranges as well as studies with additional interventions unrelated to gaze stability. Search criteria were restricted to clinical trials with human subjects ${ }^{16}$ and the English language. The concept was to examine the reporting of parameters of exercises and outcome measures used to evaluate change in gaze stability. The context was not limited and left open.

\section{Data extraction}

A Microsoft Excel (2016) spreadsheet was created to document relevant information from the articles related to dose and dosage of gaze stabilisation exercises, other interventions provided, patient diagnosis, method of diagnosis and outcomes measures related to gaze stability.

\section{Analysis and presentation of results}

All analyses were performed in Microsoft Excel (2016). Following data extraction, coding was performed to categorise findings for each of the dependent variables. Patient diagnoses consisted of seven different categories of healthy (no vestibular deficit), non-specific (no specific diagnosis provided other than vertigo or dizziness), mild traumatic brain injury (mTBI), central (central nervous 
system origin of deficit such as stroke), peripheral bilateral dysfunction, peripheral unilateral dysfunction or peripheral other. Studies were sorted based on these diagnostic criteria. Dosage parameters of duration, daily and weekly frequency and dose parameters of duration and head excursion were coded in a binary fashion of either reported (1) or not reported (0). The dose parameter of head movement speed was coded in a non-binary fashion, placed into categories of slow $\left(<120^{\circ} / \mathrm{s}\right)$, fast $\left(\geq 120^{\circ} / \mathrm{s}\right)$, per patient comfort or no reported speed. Counts of studies reporting each of the dose and dosage parameters as well as number or parameters reported within each study were then determined. Finally, outcome measures were categorised into none reported, patient-reported outcome measures (PROMs) only, clinical measures of gaze stability only, instrumented parameters of gaze stability only and those studies that used combinations of clinical, instrumented and PROMs. Again, the number of studies that used each outcome measure was then determined. Only descriptive statistics were performed in this analysis.

\section{RESULTS}

The initial search yielded 1609 studies. After duplicates were removed, a total of 1120 citations were identified for title and abstract review. Based on a review of the title and abstract, 955 articles were excluded. Overall, 165 fulltext articles were obtained and assessed for eligibility of inclusion in this study; 120 studies met the criteria. We also reviewed the references of the full-text articles and found an additional 18 references that met the inclusion criteria. A total of 138 studies were included in this review (figure 1).

Diagnoses of peripheral dysfunctions consisted of $54 \%$ of the articles including unilateral peripheral vestibular dysfunction (50 studies), bilateral peripheral vestibular

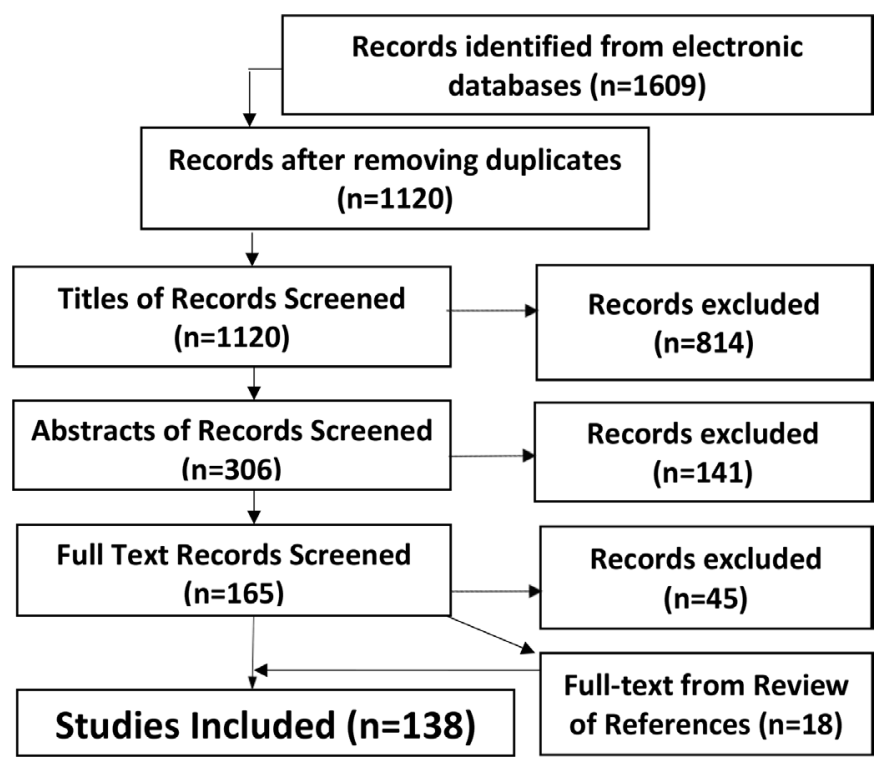

Figure 1 Flowchart for a search of electronic databases. dysfunction (31 studies) and other peripheral diagnoses such as dehiscence and cervicogenic dizziness ( 5 studies). Twenty-two studies included individuals with both unilateral and bilateral peripheral dysfunctions as separate patient populations. The results from these studies were appropriately included in the analysis of each group. Sixteen studies included individuals with diagnoses of central dysfunctions (stroke, visual vertigo, multiple sclerosis and motion sickness). Eleven studies included patients with mTBI and seven studies involved healthy subjects without vestibular deficits. In 39 of the studies, the origin of dizziness was non-specific or included a cohort of patients with many different possible origins of dysfunction. Interestingly, although gold standard evaluations of peripheral dysfunction have been established, only 26 of the 50 studies used calorics to determine a diagnosis of unilateral vestibular dysfunction, and 12 of the 32 studies addressing bilateral vestibular dysfunction used the rotatory chair method for diagnosis. Further, 55 of all 138 studies failed to describe methods of determining the diagnosis described in their study further than a subjective report of dizziness.

Data extraction revealed that only 13 studies $(9.4 \%)$ reported all six parameters of exercise prescription, including the three dosage parameters of duration, weekly frequency and daily frequency and the three dose parameters of repetition duration, exercise speed and head excursion. ${ }^{17-29}$ It is interesting to note that 9 of the 13 studies reporting all parameters of dose and dosage were studies involving very short intervention durations of either a single session or an episode of less than 2 weeks. The most common number of reported exercise parameters $(23.2 \%$ of studies) was three out of six. Interestingly, $12.3 \%$ of studies failed to report any parameters of gaze stability exercise dose or dosage. Studies reported parameters of dosage far more frequently (duration $77.3 \%$, daily frequency $69.8 \%$, weekly frequency $73.0 \%$ ) than dose (repetition duration $51.6 \%$, speed $30.2 \%$ and excursion $20.8 \%$ ) (figure 2, online supplemental appendix 2). This trend was similar across all diagnoses studied. By far the least reported was excursion of head movement during exercise, and varied from 'patient comfort' to $10^{\circ}-20^{\circ}$ in each direction. When reported, speed of exercise also varied. Several studies described the speed as patient comfort and tolerance (16 studies), predetermined set speeds (9 studies) or progressive increases to a set speed over time ( 5 studies). Exercise speeds were reported in degrees per second or as a frequency in Hz. Unfortunately, six studies that reported movement speed as $\mathrm{Hz}$ did not report head movement excursion, thus rendering an angular velocity of head movement as undetermined.

Outcome measures that specifically addressed either symptom resolution or changes in gaze stabilisation were divided into no report, PROMs, clinical evaluations and instrumented measurements (figure 3, online supplemental appendix 2). By far, the most common type of outcome used was PROMs, where $52.2 \%$ of studies used a PROM as the only measurement of change, and an 


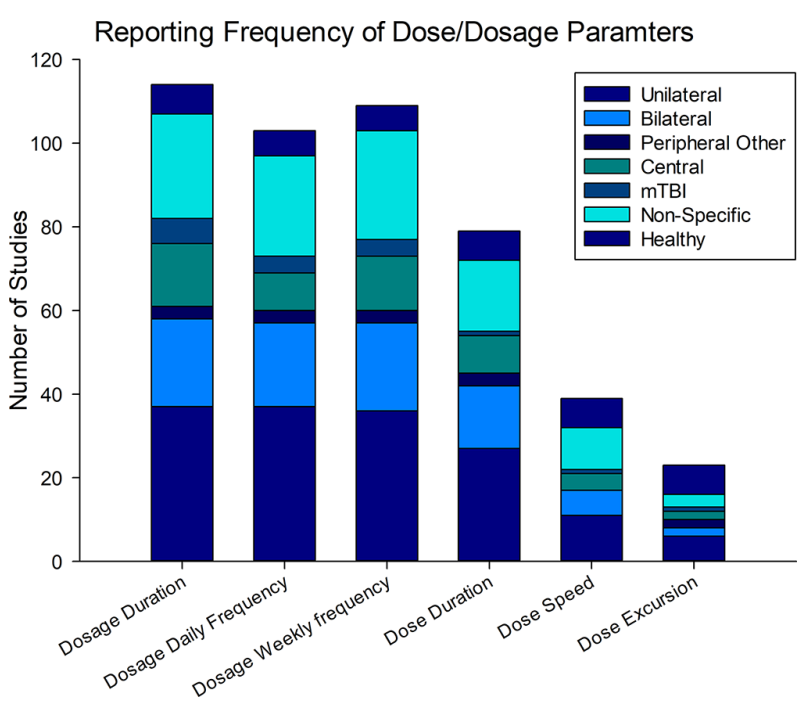

Figure 2 The number of publications that reported six different parameters of gaze stabilisation exercise dose and dosage for seven different patient populations. Numerical values are provided in online supplemental appendix 2. mTBI, mild traumatic brain injury.

additional $16.6 \%$ of studies used a PROM in combination with a clinical or instrumented measure. The Dizziness Handicap Inventory was the most commonly used PROM, reported in $52.9 \%$ of studies that used this type of instrument. A numerical rating scale of dizziness symptoms was the second most common, making up $27.1 \%$ of PROM reported. Clinical tests of gaze stability were used in $21.1 \%$ of studies, and instrumented measures were used in $14.7 \%$ of studies. The Dynamic Visual Acuity Test was the most commonly reported $(57.5 \%)$ clinical test, followed by the Head Impulse Test $(14.9 \%)$. The most commonly reported instrumented test was a measure of

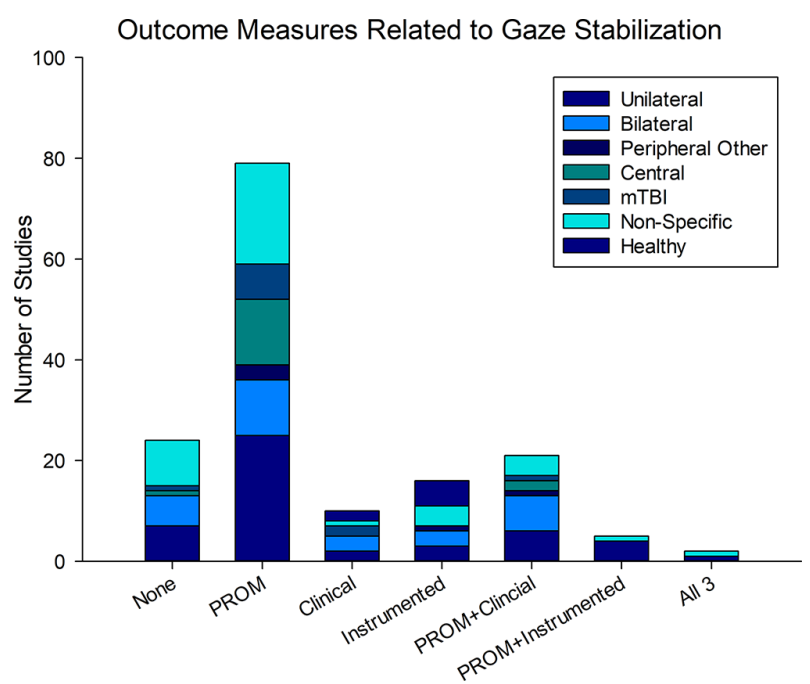

Figure 3 The number of publications that report using gaze stability outcome measures classified as clinical, instrumented or patient reported (PROM), as well as the combinations of each. Numerical values are provided in online supplemental appendix 2. mTBI, mild traumatic brain injury.
Vestibular Ocular Reflex (VOR) gain (64.7\%) through a video Head Impulse Test, followed by a computerised Dynamic Visual Acuity Test (13.3\%). Only $1.3 \%$ of studies used a combination of all three types of measures. Despite using gaze stability exercises, $14.0 \%$ of studies included no outcome measure to assess change in gaze stability or improvements in patient-reported symptoms related to gaze stabilisation as a result of their investigation.

While this scoping review emphasises reporting of exercise parameters and outcome measures of gaze stabilisation exercises, we found that 113 of the studies used other exercises in addition to gaze stabilisation. Other interventions included balance and gait exercises, general strengthening and conditioning exercises, manual therapy, cervical stretching and strengthening, visual motor exercises, habituation training and Tai Chi.

\section{DISCUSSION}

This review aimed to scope the literature to determine reporting of information related to dose and dosage of gaze stabilisation for any population receiving a gaze stabilisation intervention. We intended to document any differences in dose or dosage related to the type of dysfunction as well as capture the outcome measures being used to assess the efficacy of gaze stabilisation exercises. Gaze stabilisation exercises were used across a wide range of diagnoses including unilateral $\mathrm{VH}$, bilateral $\mathrm{VH}$, other peripheral dysfunctions, central causes, mTBI and healthy individuals. Our main findings from reviewing 138 publications that implemented gaze stabilisation interventions were: (1) only 13 studies included all gaze stabilisation dose and dosage parameters necessary for intervention replication, (2) PROMs were overwhelmingly the most common method to assess change in function and (3) the majority of studies included concurrent exercises not targeting gaze stabilisation.

Reporting of dose and dosage of an intervention is essential to be able to fully reproduce the intervention, as well as understand the effect of the intervention on outcomes. When prescribing gaze stabilisation exercises, elements of dose should include exercise duration, speed of head movement and excursion of head movement; dosage should include daily frequency, weekly frequency and length of time (eg, weeks) one should perform the exercise. Our review found large gaps in reporting of all six parameters of dosage and dose. Surprisingly, $12.3 \%$ of the studies did not report any parameters of dosage or dose for the gaze stabilisation interventions included in the study, making it impossible to replicate or translate to a clinical environment. The most commonly reported parameters were elements of dosage, while the least frequently reported parameters were dose speed and excursion. This may be due in part to the ease in which measures of dosage can be prescribed and documented. When dose speed and excursion were reported, values varied widely. Exercise velocities and frequencies ranged from $20^{\circ} / \mathrm{s}^{226}$ to $320^{\circ} / \mathrm{s}^{30}$ and from 0.04 to $10 \mathrm{~Hz}$, 
respectively. In a number of studies, the speed was often up to patient's discretion or comfort.

For the studies that did report on all aspects of dose and dosage, all but three were individual case studies and experimental paradigms that lasted less than 1 week, most often performed on healthy individuals. This could be due in part to a lack of equipment in the clinical and home setting to measure speed and/or excursion of head movement. However, our review found several different technologies being used or in development that can assist with measuring speed and excursion. ${ }^{17}{ }^{19-22} 26$ 31-33 Most involved wearable head mounted technology that might be more accessible to a clinical environment. ${ }^{19} 202231-33$ Two studies used an optokinetic training paradigm on a Digital Versatile Disc (DVD) allowing for a low-tech option of training while simultaneously controlling for all parameters. ${ }^{1726}$ Further use of technologies like these is encouraging, as it would ensure that all aspects of dose and dosage are captured and recorded to help determine the optimal parameters for gaze stabilisation exercises.

We also found gaps in the literature related to the outcome measures used to document changes in gaze stability. A substantial portion of studies (14\%) had no assessment of gaze stabilisation, despite the fact that gaze stabilisation was an intervention. We found that the most common outcome measures used to assess gaze stabilisation were PROMs. The Dizziness Handicap Inventory was the most widely used measure; however, it has no capacity to measure gaze stability in isolation. While PROMs are easy to administer, they only provide indirect assessment of gaze stabilisation through the resolution of symptoms and reported improvements in functions that incorporate gaze stability. In our opinion, using only PROMs may be problematic as it is difficult to determine if improvements on these scales are due to actual improvements in gaze stabilisation versus other possible factors, such as adapting daily activities to limit head movement and/or limiting environment complexity to limit symptoms.

Instrumented measures of gaze stability are most accurate to assess changes in VOR gain; however, only 19 studies used these means. ${ }^{34}$ This may also be due to limitations in access to equipment necessary to complete these, such as a rotatory chair. Advances in more portable technology, such as video Frenzel lenses and other wearable devices, are becoming more available and affordable. We found several studies which used video head impulse testing to determine changes in VOR function after gaze stabilisation exercises. ${ }^{20}{ }^{25} 35-37$ Technology such as this can be used to detect changes in VOR in a clinical setting and will facilitate more accurate depiction of change in gaze stability without access to a formal research laboratory or costly equipment.

Although outside the initial purpose of this scoping review, we did find that only 25 studies focused solely on gaze stabilisation as an intervention. ${ }^{19-22} 2528303233$ 38-53 Most studies included a combination of gaze stabilisation with other interventions such as balance and gait training. Although effectiveness rather than efficacy may be determined by incorporating a myriad of simultaneous interventions, further caution may be required depending on the types of exercises issued. It may be that certain exercises interfere with the effectiveness of gaze stabilisation if performed simultaneously or in sequence. Further studies are needed to determine the interaction between gaze stabilisation and other types of interventions thereby determining combinations or sequences of exercises that provide optimal recovery in gaze stabilisation and overall function.

The ultimate goal in improving the reporting of dose and dosage and use of outcome measures related to gaze stabilisation is the ability to determine the appropriate dose and dosage for individuals with vestibular dysfunction. Filling the gaps in the literature will help to determine if different dosages are needed for different types of vestibular dysfunction. While we had hoped to be able to comment on dosage and dose for different patient populations, we instead found that there is a widespread lack of reporting on the six specific parameters. Future studies should focus on reporting of all parameters of dosage and dose in order to ensure the ability to establish optimal interventions and reproducibility in the clinic and during home exercise programmes. Future studies should also include a measure of gaze stabilisation as an outcome, ideally one that is clinical or instrumented instead of patient report alone. Studies should also consider using only gaze stabilisation as an intervention to establish efficacy or to investigate the potential change in effectiveness of gaze stability exercises when performed concurrently with other types of exercises (eg, static or dynamic stability). As previously mentioned, advancements in technology that can be translated to the clinic in a costeffective manner will likely pave the way for future studies that address each of these elements.

A limitation of this review is that we restricted the search to only those articles written in English and those with full text available. Despite this, we reviewed a substantial number of articles to reach our conclusions that there is no consistency in the reporting of dosage or dose or the use of outcome measures for gaze stabilisation interventions.

\section{CONCLUSION}

Our scoping review found gaps in the literature related to reporting of dose and dosage of gaze stabilisation exercises. We also found gaps related to the use of clinical or instrumented outcome measures to document changes in gaze stabilisation. Future research should focus on controlling for and reporting on all aspects of dose and dosage and utilisation of clinical or instrumented outcome measures of gaze stability that do not rely solely on patient report. This will allow for determination of the optimal prescription of gaze stabilisation exercises. 
Acknowledgements The authors would like to thank Kendel Lipe and Ashley Warren for their contributions to the initial literature search, development of search terms and assistance with data analysis.

Contributors KRC and KG contributed to the initial conceptualisation of the review including search terms, inclusion and exclusion criteria, article reviewing and data analysis. All authors assisted with the data collection, drafting and revising of the manuscript and have approved the final version to be published and its accuracy and integrity. KRC is responsible for the overall content as guarantor.

Funding The authors have not declared a specific grant for this research from any funding agency in the public, commercial or not-for-profit sectors.

Competing interests None declared.

Patient consent for publication Not applicable.

Ethics approval This study does not involve human participants.

Provenance and peer review Not commissioned; externally peer reviewed.

Data availability statement Data are available upon reasonable request. Data include extracted information from each manuscript reviewed as detailed in the Results section. Researchers may submit a request to keithcole@gwu.edu.

Supplemental material This content has been supplied by the author(s). It has not been vetted by BMJ Publishing Group Limited (BMJ) and may not have been peer-reviewed. Any opinions or recommendations discussed are solely those of the author(s) and are not endorsed by BMJ. BMJ disclaims all liability and responsibility arising from any reliance placed on the content. Where the content includes any translated material, BMJ does not warrant the accuracy and reliability of the translations (including but not limited to local regulations, clinical guidelines, terminology, drug names and drug dosages), and is not responsible for any error and/or omissions arising from translation and adaptation or otherwise.

Open access This is an open access article distributed in accordance with the Creative Commons Attribution Non Commercial (CC BY-NC 4.0) license, which permits others to distribute, remix, adapt, build upon this work non-commercially, and license their derivative works on different terms, provided the original work is properly cited, appropriate credit is given, any changes made indicated, and the use is non-commercial. See: http://creativecommons.org/licenses/by-nc/4.0/.

\section{ORCID iD}

Keith R Cole http://orcid.org/0000-0002-3573-3859

\section{REFERENCES}

1 Grill E, Heuberger M, Strobl R, et al. Prevalence, determinants, and consequences of vestibular hypofunction. results from the KORAFF4 survey. Front Neurol 2018;9:1076.

2 Zalewski CK. Aging of the human vestibular system. Semin Hear 2015;36:175-96.

3 Rauch SD, Velazquez-Villaseñor L, Dimitri PS, et al. Decreasing hair cell counts in aging humans. Ann N Y Acad Sci 2001;942:220-7.

4 Jen JC. Bilateral vestibulopathy: clinical, diagnostic, and genetic considerations. Semin Neurol 2009;29:528-33.

5 Lee JD, Kim C-H, Hong SM, et al. Prevalence of vestibular and balance disorders in children and adolescents according to age: a multi-center study. Int J Pediatr Otorhinolaryngol 2017;94:36-9.

6 Ward BK, Agrawal Y, Hoffman HJ, et al. Prevalence and impact of bilateral vestibular hypofunction: results from the 2008 us National health interview survey. JAMA Otolaryngol Head Neck Surg 2013:139:803-10.

7 McDonnell MN, Hillier SL. Vestibular rehabilitation for unilateral peripheral vestibular dysfunction. Cochrane Database Syst Rev 2015;1:CD005397.

8 Pavlou M. The use of optokinetic stimulation in vestibular rehabilitation. J Neurol Phys Ther 2010;34:105-10.

9 Goebel JA, Tungsiripat N, Sinks B, et al. Gaze stabilization test: a new clinical test of unilateral vestibular dysfunction. Otol Neurotol 2007:28:68-73.

10 Whitney SL, Alghwiri A, Alghadir A. Physical therapy for persons with vestibular disorders. Curr Opin Neurol 2015;28:61-8.

11 Han BI, Song HS, Kim JS. Vestibular rehabilitation therapy: review of indications, mechanisms, and key exercises. J Clin Neurol 2011;7:184-96

12 Hall CD, Herdman SJ, Whitney SL, et al. Vestibular rehabilitation for peripheral vestibular hypofunction: an evidence-based clinical practice guideline: from the American physical therapy association Neurology section. J Neurol Phys Ther 2016;40:124-55.
13 Tricco AC, Lillie E, Zarin W, et al. PRISMA extension for scoping reviews (PRISMA-ScR): checklist and explanation. Ann Intern Med 2018;169:467-73.

14 Arksey H, O'Malley L. Scoping studies: towards a methodological framework. Int J Soc Res Methodol 2005;8:19-32.

15 Peters MDJ GC, Mclnerney P, Munn Z, et al. Chapter 11: Scoping Reviews (2020 version). In: Aromataris E, Munn Z, eds. Joanna Briggs Institute Reviewer's Manual. JBI, 2020. https:// reviewersmanual.joannabriggs.org/

16 Health Nlo. Notice of Revised NIH Definition of "Clinical Trial". grants. nih.gov. Office of Science Policy, 2014.

17 Manso A, Ganança MM, Caovilla HH. Vestibular rehabilitation with visual stimuli in peripheral vestibular disorders. Braz J Otorhinolaryngol 2016;82:232-41.

18 Horning E, Gorman S. Vestibular rehabilitation decreases fall risk and improves gaze stability for an older individual with unilateral vestibular hypofunction. J Geriatr Phys Ther 2007;30:121-7.

19 Migliaccio AA, Schubert MC. Pilot study of a new rehabilitation tool: improved unilateral short-term adaptation of the human angular vestibulo-ocular reflex. Otol Neurotol 2014;35:e310-6.

20 Rinaudo CN, Schubert MC, Cremer PD, et al. Improved oculomotor physiology and behavior after unilateral incremental adaptation training in a person with chronic vestibular hypofunction: a case report. Phys Ther 2019;99:1326-33.

21 Schubert MC, Della Santina CC, Shelhamer M. Incremental angular vestibulo-ocular reflex adaptation to active head rotation. Exp Brain Res 2008;191:435-46.

22 Todd CJ, Hubner PP, Hubner P, et al. StableEyes-A portable vestibular rehabilitation device. IEEE Trans Neural Syst Rehabil Eng 2018;26:1223-32.

23 Kramer PD, Shelhamer M, Zee DS. Short-Term adaptation of the phase of the vestibulo-ocular reflex (VOR) in normal human subjects. Exp Brain Res 1995;106:318-26.

24 Mahfuz MM, Schubert MC, Figtree WVC, et al. Optimal human passive vestibulo-ocular reflex adaptation does not rely on passive training. J Assoc Res Otolaryngol 2018;19:261-71.

25 Migliaccio AA, Schubert MC. Unilateral adaptation of the human angular vestibulo-ocular reflex. J Assoc Res Otolaryngol 2013;14:29-36.

26 Pavlou M, Bronstein AM, Davies RA. Randomized trial of supervised versus unsupervised optokinetic exercise in persons with peripheral vestibular disorders. Neurorehabil Neural Repair 2013;27:208-18.

27 Rossi-Izquierdo M, Santos-Pérez S, Soto-Varela A. What is the most effective vestibular rehabilitation technique in patients with unilateral peripheral vestibular disorders? Eur Arch Otorhinolaryngol 2011;268:1569-74.

28 Shelhamer M, Tiliket C, Roberts D, et al. Short-Term vestibuloocular reflex adaptation in humans. II. error signals. Exp Brain Res 1994:100:328-36.

29 Tiliket C, Shelhamer M, Roberts D, et al. Short-Term vestibulo-ocular reflex adaptation in humans. Exp Brain Res 1994;100:316-27.

30 Sadeghi NG, Sabetazad B, Rassaian N, et al. Rebalancing the vestibular system by unidirectional rotations in patients with chronic vestibular dysfunction. Front Neurol 2018:9:1196.

31 Bhatti PT, Herdman SJ, Roy SD, et al. A prototype Head-Motion monitoring system for in-home vestibular rehabilitation therapy. $J$ Bioeng Biomed Sci 2011;1.

32 Crane BT, Schubert MC. An adaptive vestibular rehabilitation technique. Laryngoscope 2018;128:713-8.

33 Gimmon Y, Migliaccio AA, Kim KJ, et al. Vor adaptation training and retention in a patient with profound bilateral vestibular hypofunction. Laryngoscope 2019;129:2568-73.

34 Starkov D, Strupp M, Pleshkov M, et al. Diagnosing vestibular hypofunction: an update. J Neurol 2021;268:377-85.

35 Navari E, Cerchiai N, Casani AP. Assessment of vestibulo-ocular reflex gain and catch-up saccades during vestibular rehabilitation. Otol Neurotol 2018;39:e1111-7.

36 Micarelli A, Viziano A, Micarelli B, et al. Vestibular rehabilitation in older adults with and without mild cognitive impairment: effects of virtual reality using a head-mounted display. Arch Gerontol Geriatr 2019;83:246-56

37 Loyd BJ, Fangman A, Peterson DS, et al. Rehabilitation to improve gaze and postural stability in people with multiple sclerosis: study protocol for a prospective randomized clinical trial. BMC Neurol 2019;19:119.

38 Bayat A, Saki N. Effects of vestibular rehabilitation interventions in the elderly with chronic unilateral vestibular hypofunction. Iran J Otorhinolaryngol 2017;29:183-8.

39 Braswell J, Rine RM. Preliminary evidence of improved gaze stability following exercise in two children with vestibular hypofunction. Int $J$ Pediatr Otorhinolaryngol 2006;70:1967-73. 
40 Chen P-Y, Hsieh W-L, Wei S-H, et al. Interactive wiimote gaze stabilization exercise training system for patients with vestibular hypofunction. J Neuroeng Rehabil 2012;9:77.

41 Cohen H, Kane-Wineland M, Miller LV, et al. Occupation and visual/ vestibular interaction in vestibular rehabilitation. Otolaryngol Head Neck Surg 1995;112:526-32.

42 Cohen HS. Disability and rehabilitation in the dizzy patient. Curr Opin Neurol 2006;19:49-54.

43 Cohen HS, Kimball KT. Increased independence and decreased vertigo after vestibular rehabilitation. Otolaryngol Head Neck Surg 2003;128:60-70.

44 Cohen HS, Kimball KT. Changes in a repetitive head movement task after vestibular rehabilitation. Clin Rehabil 2004;18:125-31.

45 Enticott JC, O'leary SJ, Briggs RJS. Effects of vestibulo-ocular reflex exercises on vestibular compensation after vestibular schwannoma surgery. Otol Neurotol 2005;26:265-9.

46 Gizzi M. The efficacy of vestibular rehabilitation for patients with head trauma. Journal of Head Trauma Rehabilitation 1995;10:60-77.
47 Meli A, Zimatore G, Badaracco C, et al. Vestibular rehabilitation and 6-month follow-up using objective and subjective measures. Acta Otolaryngol 2006;126:259-66.

48 Moaty AS, EL Mahallawi TH, Emara AA, et al. The role of customized vestibular rehabilitation with visual desensitization in the management of visual vertigo syndrome. Hearing Balance and Communication 2017:15:127-32.

49 Montfoort I, Van Der Geest JN, Slijper HP, et al. Adaptation of the cervico- and vestibulo-ocular reflex in whiplash injury patients. $J$ Neurotrauma 2008;25:687-93.

50 Pfaltz CR. Vestibular compensation. physiological and clinical aspects. Acta Otolaryngol 1983;95:402-6.

51 Rine RM, Wiener-Vacher S. Evaluation and treatment of vestibular dysfunction in children. NeuroRehabilitation 2013;32:507-18.

52 Viirre E, Draper M, Gailey C, et al. Adaptation of the VOR in patients with low VOR gains. J Vestib Res 1998;8:331-4.

53 Viirre E, Sitarz R. Vestibular rehabilitation using visual displays: preliminary study. Laryngoscope 2002;112:500-3. 